\title{
OPTIMIZED CLASS-SEPARABILITY IN HYPERSPECTRAL IMAGES
}

\author{
Sumera Sattar ${ }^{1}$, Haris Ahmad Khan ${ }^{2}$, Khurram Khurshid ${ }^{1}$ \\ ${ }^{1}$ EE Department, Institute of Space Technology, 1 Islamabad highway, Islamabad, 44000, Pakistan \\ ${ }^{2}$ Laboratoire Electronique, Informatique et Image (Le2i), Université de Bourgogne, Dijon, France \\ ${ }^{1}$ \{ sumera, khurram.khurshid\}@ ist.edu.pk, ${ }^{2}$ Haris-Ahmad.Khan@u-bourgogne.fr
}

\begin{abstract}
Image visualization techniques are mostly based on three bands as RGB color composite channels for human eye to characterize the scene. This, however, is not effective in case of hyper-spectral images (HSI) because they contain dozens of informative spectral bands. To eliminate redundancy of spectral information among these bands, dimensionality reduction (DR) is applied while at the same trying to retain maximum information. In this paper, we propose a new method of information-preserved hyperspectral satellite image visualization that is based on fusion of unsupervised band selection techniques and color matching function (CMF) stretching. The results show consistent, edge-preserved and pre-attentive feature less images with high class separability. Different visualization techniques are compared to demonstrate the effectiveness of our scheme that can prompt an important advancement in the field.
\end{abstract}

Index Terms - Dimensionality reduction, hyperspectral image visualization, class separability

\section{INTRODUCTION}

Hyper-spectral images contain wide range of spectral information in adjacent narrow bands. However, as three bands are used in display systems, rendering hyper-spectral images directly on conventional display systems is not feasible. HSI are used in various applications $[1,2]$ thus having capability of a good meaningful visualization is a prime requirement. Fundamentally there can be two ways to display HS data. First, based on classification where we display each class with different color thus resulting in a good but unnatural looking image. The second is to map $\mathrm{N}$ components to three for display but it results in loss of information. Band selection techniques for HS image visualization are categorized into supervised and unsupervised methods. Supervised methods like minimum estimated abundance covariance (MEAC) depend on spectral signature of data [4]. On the other hand, unsupervised methods do not rely on any prior knowledge but they are computationally expensive. For example, linear combination of the data has been utilized to acquire three new bands in method called Principal component analysis (PCA) [5]. But in certain cases, its performance drops down due to badly correlated data. Therefore, a well-organized and suitable means of visualization of the HSI is needed [3].

A lot of work has been done in fusion of data. Some bands are highly informative during fusion and weak features are suppressed. Recently saliency based visualization (SBV) of HSI method has been proposed [17] in which spatial and spectral information about all bands was taken into account, but this approach doesn't possess class separability property. So an efficient, low complexity, computationally efficient, highly informative and more class distinctive method are required. To address the previously stated issues and to make utilization of this rich source of data, we propose a new HSI visualization technique by trying to overcome the problems using different useful band selection techniques.

The rest of this paper is arranged as follows: different band selection systems are presented in section 2 . In section 3 proposed visualization approaches is presented with two schemes/variations. The experimental results are presented in section 4 .

\section{LITERATURE REVIEW}

The visualization using bilateral filtering is based on calculating the weight of each pixel in band, the weight of each pixel in whole HSI is individually calculated, depending on the relative contribution of the pixel in the image [6]. One bit transforms (1BT) method is investigated based on using all bands of HSI data for selecting the appropriate bands for RGB show, determination of bands is performed completely over 1BT representations by means of typically Boolean operation [7]. The segmented principle components transformation (PCT) is the fusion method, three methods for dividing the bundle of bands into subgroups is presented. First principle component of every subgroup is utilized for display [8]. A Pixel-level fusion [9] is proposed based on perceptual attributes that display an image with perceptually high quality. Typical DR methods to display RGB image principle components analysis is 
investigated [10], [11]. Noise-adjusted principal component analysis (NAPCA) is performed in two steps. The first step is noise whitening and other step is to perform ordinary PCA to the noise-whitened data because of the unity of the noise whitened data in noise variance [12]. Other transformation-based color display approaches are proposed by Jacobson et al [13], [14], where to produce naturallooking images fixed weighting linear spectral envelopes are used that displays the same materials in different data sets consistently but they will be incapable to show some different objects distinctively because there is no adaptation to specific image information. Hyvarinen et al. [15] proposed the idea to decompose a group of multivariate signals into statistically independent component sources with minimum loss of object information. Wang et al [16] investigated DR-ICA that can capture information using virtual dimensionality to approximate the quantity of dimensions needed. Saliency based visualization [17] Investigated weighted fusion method by calculating the saliency in each band of HSI. Both spatial and spectral information are taking into account. They applied the saliency map technique on PCA image by dividing data into three equal parts. For final visualization fused PCA, CMF and saliency based tri-color images. But these techniques don't ensure the different class information in the final image. Supervised band selections methods are used for visualization e.g. Minimum estimated abundance covariance (MEAC) [18] depend on spectral signature and many other mentions in [19].

\section{METHODOLOGY}

In this paper, we investigate a new methodology for displaying HS image considering two major goals: class separability and consistent rendering [14]. To accomplish these objectives, it is essential to discover effective information handling steps. We selected three unsupervised band selection methods on the basis of their effectiveness in remote sensing that include Principal Component Analysis (PCA) [5], Maximum Noise Fraction (MNF) [20] and Independent Component Analysis (ICA) [16]. The goal here is to show significant information as distinctive as possible for high-class separability. PCA uses highest Eigen values to select the three principle components from hundreds of band. In MNF [20] goal is to pick bands in a way that maximize their signal to-noise (SNR) ratio by minimizing the noise fraction using a linear transformation. MNF considers the spatial information of each band for covariance matrix that makes MNF much more effective and reliable to image quality.

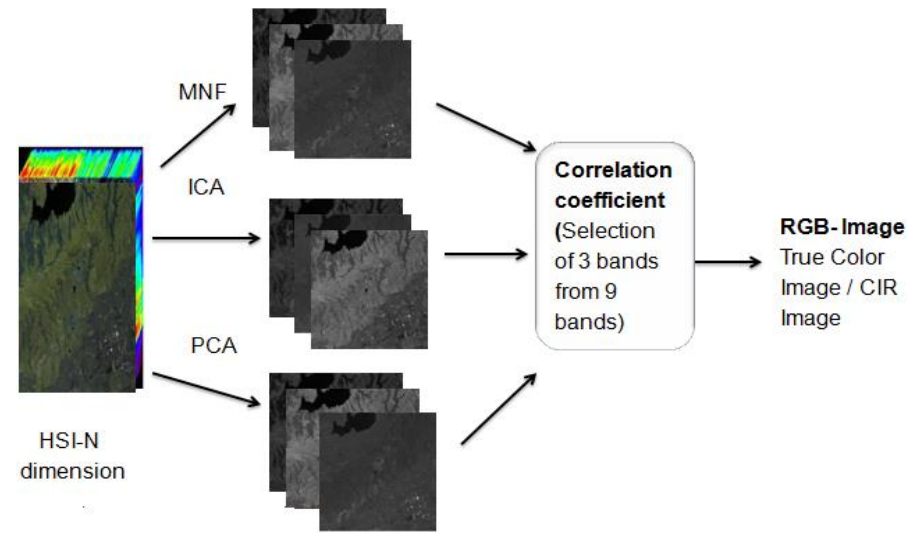

Fig 1: Scheme-I (a) True Color (b) CIR Display

The third method used is ICA [15] that measures data, statistical independency using mutual information as a criterion. It captures data that can't be retained or preserved by second-order statistics based dimension reduction methods like PCA and MNF. These three methods are applied in 2 ways:

\subsection{Scheme-I: a) True Color b) CIR}

This scheme is designed in two ways namely true color and near infrared color. Here the original HSI is segmented into a) visible range (R, G, B) and b) near infrared (R, G, NIR). Then we apply three DR methods to each spectrum. From 9 selected bands further 3 uncorrelated bands are selected for tri-stimulus composite display. The resultant image for true color gives the natural appearance while CIR version is helpful to display vegetation in red color. Flowchart of scheme-I is shown in Fig.1.

\subsection{Scheme-II}

In scheme-I we selected only specific spectrum for visualization which results is loss of important information present in other bands. In this method we use all bands for image visualization. Keeping focus on our goals, first we spectrally segment the original data cube into three groups: visible range, NIR and middle infrared. In order to get highly informative featured bands, we further sub-group each spectral group into three equal parts and applied PCA on each sub-group to get principle components (PC). Through correlation metric Eq. (1) [19] from all PC's we selected the three most distinct, highly structured maximum energy bands.

$$
W\left(b_{1}, b_{2}, b_{3}\right)=\frac{S_{1}\left(b_{1}\right)+S_{2}\left(b_{2}\right)+S_{3}\left(b_{3}\right)}{S\left(b_{1}, b_{2}, b_{3}\right)}
$$



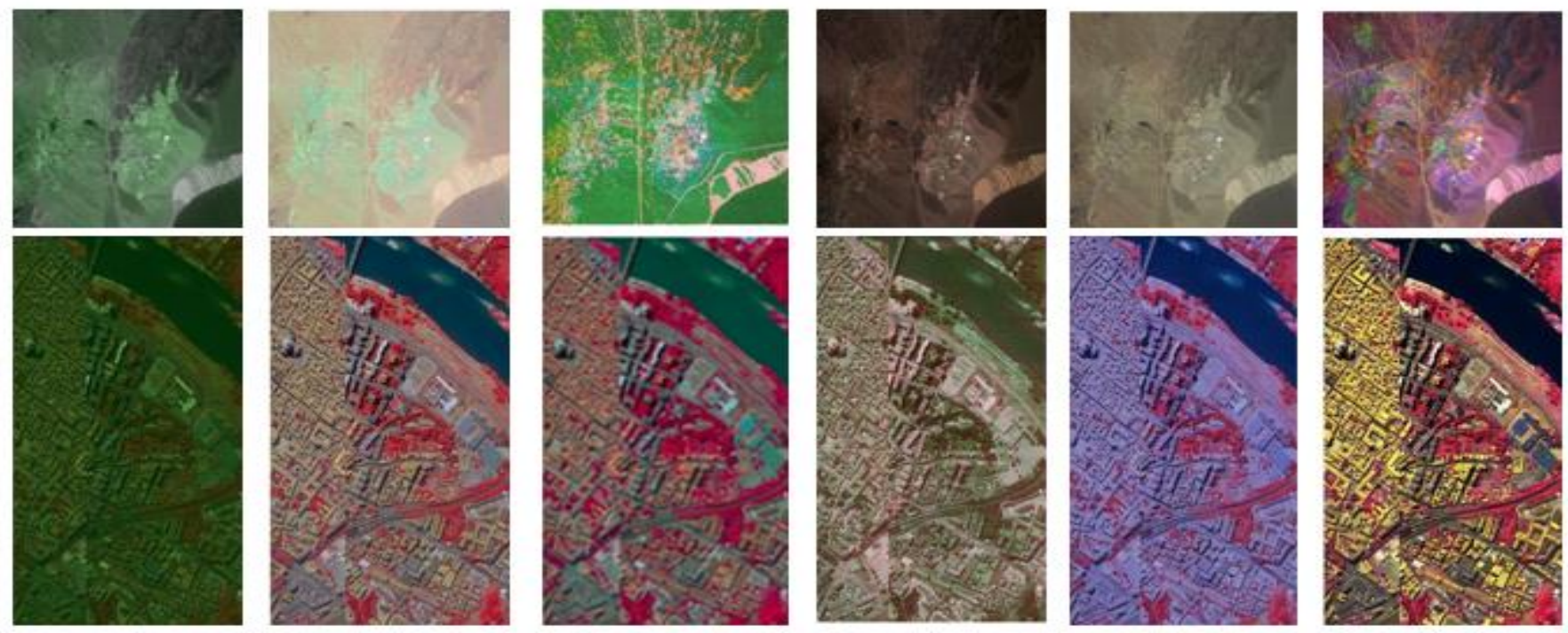

Fig.2: Row-wise: AVIRIS Cuprite, ROSIS Pavia Centre

Column from left to right: Segmented PCA [7], CMF Stretched, SBV, Scheme1a, Scheme 1b, Scheme 2 Optimized Class Separability

Bands selected in different spectral regions provide users the ability to discriminate objects. To get more distinct class information we applied separately MNF and ICA on original HSI. For MNF we select three uncorrelated bands Eq. (1) from top high SNR ranked bands. Then fusion of all RGB composite images is done to get a final image $f(m, n)$ Eq. (2) and Eq. (3).

$$
\mathrm{I}(\mathrm{m}, \mathrm{n})=\frac{P C A+I C A+M N F}{3}
$$

By adding MNF, PCA, ICA images, the fused image $\mathrm{I}(\mathrm{m}, \mathrm{n})$ gives inconsistent visualization. We also apply CMF stretching on original HSI to getcmf $(m, n)$.

$$
f(m, n)=W_{1} \cdot \mathrm{I}(\mathrm{m}, \mathrm{n})+W_{2} \cdot \mathrm{cmf}(\mathrm{m}, \mathrm{n})
$$

On the basis of Perceptual based class separability, we prefer to assign different weights (found empirically) for final tri-stimulus display. Resultant image $f(m, n)$ has the ability to discriminate different class data. The proposed approach gives image with maximum achievable distinction in the RGB components and therefore, enhanced illustration and visual discrimination of end-members.

\section{RESULTS AND DISCUSSION}

For performance evaluation, we select 3 statistical assessment parameters given in [6] where Entropy describes amount of information in image calculated as Eq.(4). Here $p x$ is the probability density of the intensity level $x$ in the image.

$$
H_{X}=-\sum_{x} p x(x) \ln p x(\mathrm{x})
$$

Mean describe average gray value and Variance $\sigma^{2}=$ $\operatorname{var}(I)$ measures the deviation of gray values of the pixels from the image mean which describes image contrast. After removing water absorption atmospheric effected bands, we performed the experiments on AVIRIS Cuprite (C) and PAVIA CENTER $(\mathrm{P})$ and compared the result with different previous methods. First image data was collected over the Cuprite mining site where the five minerals of interest are exposed alunite (A), buddingtonite (B), calcite (C), kaolinite $(\mathrm{K})$, and muscovite (M) labeled in Fig. 3(a). For better subjective assessment of minerals we zoom-in our result in Fig.3 (b) that clearly shows different class data with different contrast which is not seen in any previous methodology.

Table 1: Quantitative Results for Cuprite (C) and Pavia (P)

\begin{tabular}{|c|c|c|c|c|c|c|}
\hline Method & \multicolumn{2}{|c|}{ Entropy } & \multicolumn{2}{c|}{ Variance } & \multicolumn{2}{c|}{ Mean } \\
\hline & C & P & C & P & C & P \\
\hline SPCA & 6.73 & 6.46 & 633.8 & 434.2 & 96.33 & 98.55 \\
\hline CMF & 6.31 & 7.28 & 670.5 & 820.0 & 78.53 & 75.76 \\
\hline SBV & 7.15 & 7.32 & 594.4 & 794.4 & 95.00 & 90.87 \\
\hline Scheme-I(a) & 6.42 & 7.01 & 534.3 & 598.34 & 93.23 & 95.32 \\
\hline Scheme-I(b) & 6.64 & 7.11 & 523.8 & 543.69 & 81.25 & 87.04 \\
\hline Scheme-II & 7.45 & 7.67 & 920.2 & 849.5 & 98.37 & 99.55 \\
\hline
\end{tabular}




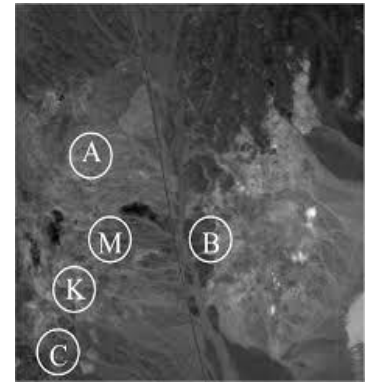

(a)

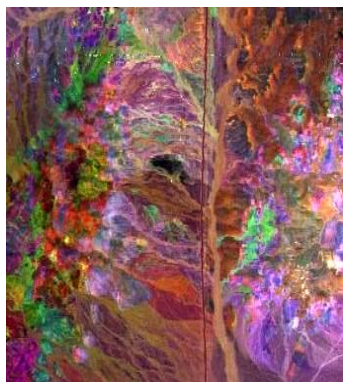

(b)
Fig.3: (a) Spatial position of five minerals [15]

(b) Result by proposed scheme II

Qualitative results are given in Fig.2 for 6 different methods ( 3 proposed and 3 state of the art). We can see that none of the images is able to discriminate the different class information present in ROSIS Pavia center image except of our proposed method (scheme- II) that displays image with special contrast, while other methods are not able to give such details. As original data contain vegetation our CIR scheme displays it in red color because infrared range is sensitive for vegetation. Quantitative assessment in Table-1 well proves that our results are highly informative as images with high variance have better contrast and appealing for visualization.

\section{REFERENCES}

[1] D. Stein, J. Schoonmaker, and E. Coolbaugh, "Hyperspectral imaging for intelligence, surveillance, and reconnaissance," Space Naval Warfare Syst. Center, San Diego, CA, Aug. 2001.

[2] J. Ardouin, J. Lávesque, and T. Rea, "A demonstration of hyperspectral image exploitation for military applications," in Proc. 10th Int. Conf. Inf.Fusion, Québec City, QC, Canada, Jul. 2007, pp. 1-8.

[3] R. A. Schowengerdt,"Remote Sensing-Models and Methods for Image Processing", 3rd ed. New York: Academic, 2007.

[4] Huqqani, Ilyas A., and Khurram Khurshid. "Comparative Study of Supervised Classification of Urban Area Hyperspectral Satellite Imagery" Journal of Space Technology, vol. 4, no. 1, pp. 7-14, July 2014

[5] X. Jia and J. A. Richard."Segmented principal components transformation for efficient hyperspectral remote-sensing image display and classification,"IEEE Trans. Geosci. Remote Sens., vol. 37, no. 1, pp. 538-542, Jan. 1999.

[6] K.Kotwal, S.Chaudhuri.Visualization of Hyperspectral Images Using Bilateral Filtering. IEEE Trans. Geosci. Remote Sensing, vol. 48, no. 5, pp. 2308-2316, May 2010.
[7] B. Demir, A. Celebi, and S. Erturk,"A low-complexity approach for the color display of hyperspectral remote-sensing images using one-bit transform-based band selection," IEEE Trans. Geosci. Remote Sens.,vol. 47, no. 1, pt. 1, pp. 97-105, 2009.

[8] V. Anastassopoulos, and GA Lampropoulos V. Tsagaris, "Fusion of hyperspectral data using segmented pct for color representation and classification," IEEE Trans. Geosci. Remote Sens.,, vol. 43, no. 10, pp. 2365-2375, 2005.

[9] V. Tsagaris and V. Anastassopoulos, "Multispectral image fusion for improved RGB representation based on perceptual attributes," Int. J. Remote Sens., vol. 26, no. 15, pp. 32413254, Aug. 2005.

[10] J. S. Tyo, A. Konsolakis, D. I. Diersen, and R. C. Olsen, "Principal components-based display strategy for spectral imagery," IEEE Trans.Geosci. Remote Sens., vol. 41, no. 3, pp. 708-718, Mar. 2003.

[11] A. Green, M. Berman, P. Switzer, and M. D. Craig, "A transformation for ordering multispectral data in terms of image quality with implications for noise removal," IEEE Trans. Geosci. Remote Sens., vol. 26, no. 1,pp. 65-74, Jan. 1988.

[12] R. E. Roger, "A faster way to compute the noise-adjusted principal components transform matrix," IEEE Trans. Geosci. Remote Sens., vol. 32,no. 6, pp. 1194-1196, Jun. 1994.

[13] N. P. Jacobson andM. R. Gupta, "Design goals and solutions for display of hyperspectral images," IEEE Trans. Geosci. Remote Sens., vol. 43, no. 11,pp. 2684-2693, Nov. 2005.

[14] N. P. Jacobson, M. R. Gupta, and J. B. Code, "Linear fusion of image sets for display," IEEE Trans. Geosci. Remote Sens., vol. 45, no. 10, pp. 3277-3288, Oct. 2007.

[15] Hyvärinen A., J. Karhunen, and E. Oja, "Independent Component Analysis"..New York: Wiley-Interscience, 2001

[16] J.Wang ,CI. Chang,"Independent Component Analysis-Based Dimensionality Reduction with Applications in Hyperspectral Image Analysis" IEEE Trans. Geosci. Remote Sens., vol. 44, no. 6, pp. 1586 - 1600, june. 2006.

[17] H.Ahmad Khan, M. Murtaza Khan.,K Khurshid, J Chanussot,. "Saliency Based Visualization of Hyper-Spectral Images" IEEE Trans. Geosci. Remote Sens.Symposium, pp.1096 - 1099, july.2015.

[18] H. Yang, Q. Du, H. Su, and Y. Sheng, "An efficient method for supervised hyperspectral band selection," IEEE Geosci. Remote Sens. Lett.,vol. 8, no. 1, pp. 138-142, 2011.

[19] H.Su, Q. Du, and P. Du, "Hyperspectral Image Visualization Using Band Selection," IEEE J. Sel. Topics Earth Observ. Remote Sens., vol. 7, no. 3, pp. 2647 - 2658, Aug 2013.

[20] A. A. Green, M. Berman, P. Switzer, and M. D. Craig, "A transformation for ordering multispectral data in terms of image quality with implications for noise removal," IEEE Trans. Geosci. Remote Sens., vol. 26, no.1, pp. 65-74, Jan. 1988. 